\title{
Refractive Collimating Microlens Arrays by Surface Tension Self-Assembly
}

\author{
Richard R. A. Syms, Member, IEEE
}

\begin{abstract}
A new process for fabrication of refractive collimating microlens arrays is demonstrated. The lens mount is formed in the bonded layer of a bonded silicon-on-insulator wafer, and rotated out of plane by the surface tension obtained by melting rectangular pads of thick photoresist. The lenses are formed by melting circular pads of the same resist. Correct alignment of the mount normal to the substrate is achieved by sequential self-assembly of a supporting frame.
\end{abstract}

Index Terms-MEMS, microlens, microoptics, self-assembly.

$\mathbf{L}$ ENSES are needed for beam collimation in microoptoelectro-mechanical systems (MOEMS) [1]. The lenses must often be set up normal to beams travelling parallel to the substrate. Fabrication involves in-plane patterning of a surface micromachined structure, and out-of-plane rotation and fixing. Fresnel lenses have been formed by etching polysilicon [2], while refractive lenses have been formed by melting photoresist [3] using techniques developed for fixed lens arrays [4].

Assembly has been performed either using an external manipulator, or using a microengine such as a linear vibromotor. Surface tension self-assembly is an alternative method, which has the potential for mass-parallel assembly of MOEMS [5]. Recently, a simple two-mask process has been developed, based on bonded silicon-on-insulator (BSOI). The mechanical parts are formed in the bonded layer, and rotation is powered by melting pads of thick resist linking fixed and moving parts [6].

In this paper, we show how surface tension self-assembly can be extended to allow the formation of out-of-plane photoresist microlens arrays on silicon frames. Fig. 1 shows the structure used, before assembly. There are three moving parts: the left- and right-hand halves of a support frame (FRAME-L and FRAME-R), and the lens mount (MOUNT). The movable parts are formed by deep reactive ion etching through the 5- $\mu$-m-thick bonded silicon layer of a BSOI wafer, and are perforated with 4 $\mu \mathrm{m}$ square holes on a $20-\mu \mathrm{m}$-pitch, so they may be freed by wet etching a $2-\mu-\mathrm{m}$ layer of $\mathrm{SiO}_{2}$ beneath. The fixed and moving parts are linked by $250 \times 40-\mu \mathrm{m}$ pads of Hoechst AZ4562 resist with a thickness of $\approx 12 \mu \mathrm{m}$. Before sacrificial etching, the resist is pre-melted at $100{ }^{\circ} \mathrm{C}$ to improve adhesion.

Assembly is performed by re-melting at $145^{\circ} \mathrm{C}$, in the sequence of Fig. 2. The surface tension torque causes FRAME-L and FRAME-R rotate in opposite directions through $45^{\circ}$, at which point a mechanical limiter prevents further rotation. This

Manuscript received April 26, 2000; revised July 20, 2000. This work was supported by EPSRC.

The author is with Department of Electrical and Electronic Engineering, Imperial College, Exhibition Road, London, SW7 2BT, U.K. (e-mail r.syms@ic.ac.uk).

Publisher Item Identifier S 1041-1135(00)09601-4.

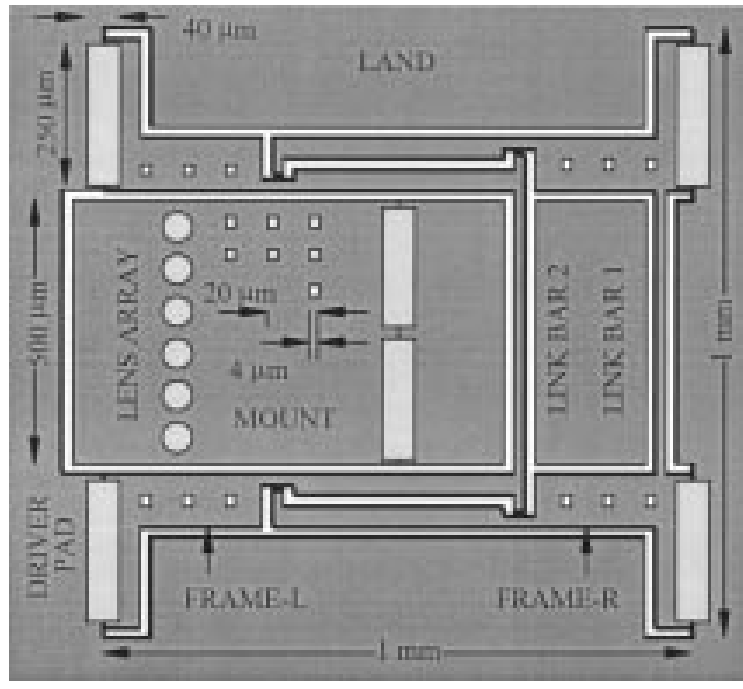

Fig. 1. Overall layout of a surface tension self-assembled microlens array.

operation establishes LINK BAR2 as a "stop" in mid-air above the substrate. MOUNT also rotates, but the mid-air stop prevents further movement after it has rotated through $90^{\circ}$. Assembly is sequential, because MOUNT must rotate through a larger angle than FRAME-L and FRAME-R, but the timings are not crucial; the stop must merely be completed before MOUNT reaches it.

The lenses are arranged in six-element arrays on an $80-\mu \mathrm{m}$ pitch, and have been successfully formed from circular pads of the thick resist in diameters up to $60 \mu \mathrm{m}$. Each element is placed over a smaller circular opening in MOUNT, to allow a 5- $\mu \mathrm{m}$ peripheral land. After lithography, the lens dies are short cylinders, whose upper surfaces are made spherical by pre-melting. After sacrificial etching, the lenses are plano-convex. However, during assembly, they are re-melted into suspended drops, constrained only by a circumferential land. The lower surfaces then also become spherical, so that the final lenses are bi-convex.

Fig. 3(a) shows the front of a completed collimator, showing that the structure has assembled correctly. Fig. 3(b) shows the rear of two lenses, highlighting their spherical surfaces, and Fig. 3(c) a top view, confirming their bi-convex profile. Fig. 4 shows quantitative data for the shape of a $60-\mu \mathrm{m}$-diameter lens, obtained by digitizing an enlarged SEM photograph. Two best-fit parabolae to the solid data points are also shown. By differentiating these parabolae twice, the radii of curvature of the surfaces were estimated as $43.9 \mu \mathrm{m}$ (upper) and $45.8 \mu \mathrm{m}$ (lower). The close agreement between these values suggests that the lens shape stabilizes when the Laplace pressure ( $p=2 \gamma / r$, where $\gamma$ is the surface tension coefficient and $r$ the radius of curvature) is equal everywhere. 

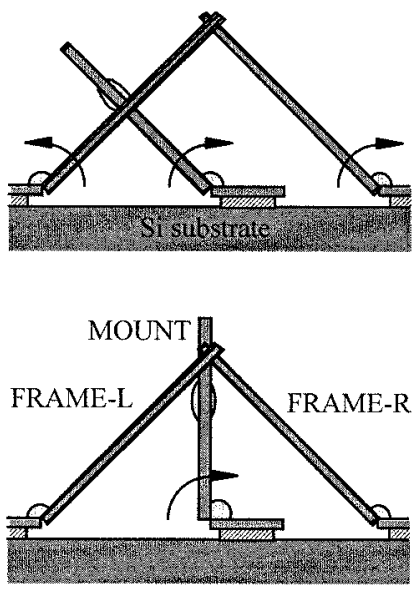

Fig. 2. Sequence of the self-assembly process.

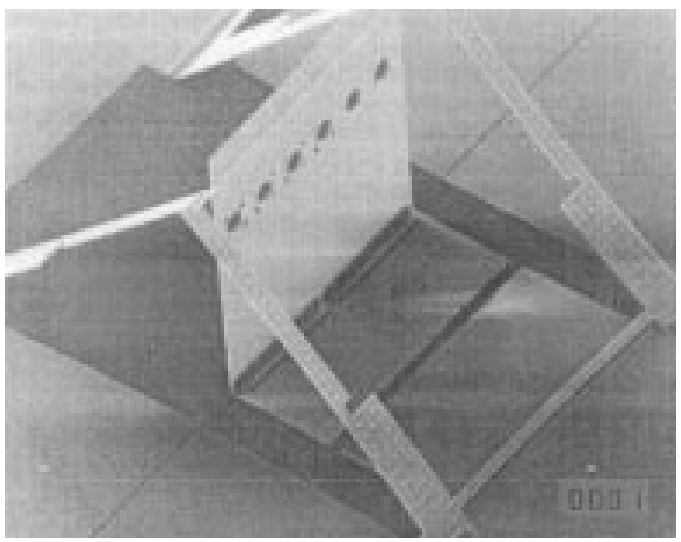

(a)

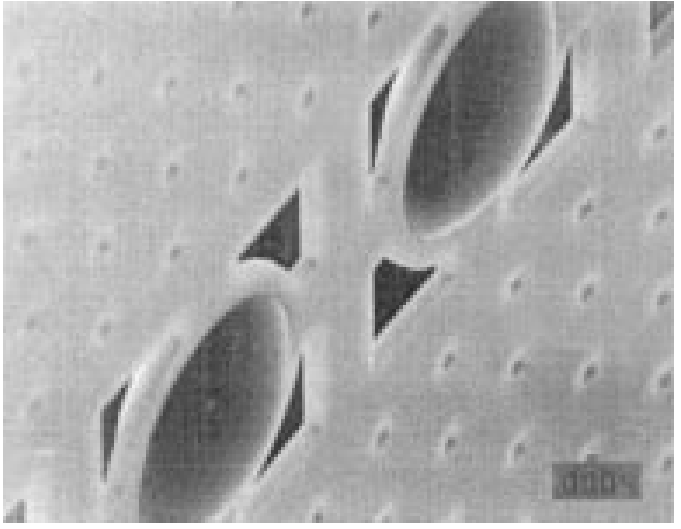

(b)

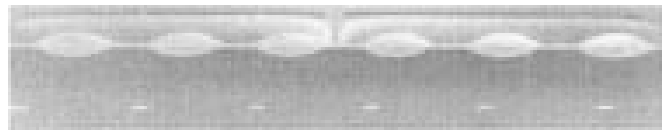

(c)

Fig. 3. SEM views of front (a), rear (b) and top (c) of an assembled microlens array.

The volume of resist in the final structure was estimated by summing three contributions (from the upper and lower spherical regions, marked I and II in Fig. 4, and from the cylindrical disc encased inside the silicon frame, marked III). The first two

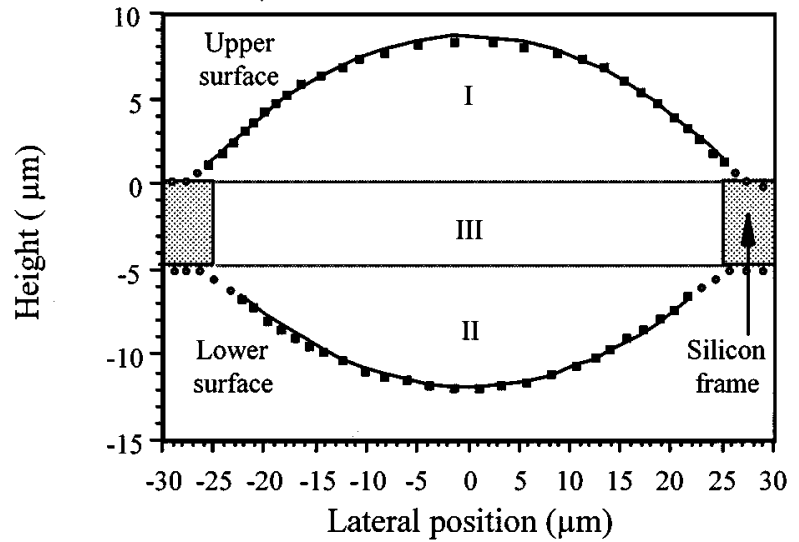

Fig. 4. Profile of a $60-\mu \mathrm{m}$-diameter lens. Points are data; lines are best-fit to parabolae.

contributions were estimated from the well-known formula for the volume of a semi-spherical droplet

$$
V=\pi r^{3}\left\{2 / 3-\cos (\theta)+\cos ^{3}(\theta) / 3\right\}
$$

where

$r \quad$ radius of the droplet;

$w \quad$ diameter of its land;

$r \quad=w /\{2 \sin (\theta)\}$.

The final volume thus obtained was found to be a factor $\eta_{v} \approx$ 0.75 of the corresponding value estimated by summing contributions from the initial cylindrical resist pad and the interior cylindrical disc, implying that shrinkage of the resist had occurred during processing. Separate diagnostic experiments were therefore performed on uniform layers of AZ4562. After melting at $145^{\circ} \mathrm{C}$ for $30 \mathrm{~min}$, the resist thickness reduced to $\eta_{L} \approx 0.9$ of its initial value. This shrinkage corresponds to $\eta_{v}=\eta_{L}^{3} \approx 0.73$, in good agreement with the above.

The focal length of the microlenses were measured in symmetric fiber-lens-fiber coupling experiments performed with $8 / 125 \mu \mathrm{m}$ telecommunications fiber at $\lambda=0.633 \mu \mathrm{m}$. For example, Fig. 5 shows the variation of throughput with fiber-fiber separation $\mathrm{s}$ for a $40-\mu \mathrm{m}$-diameter lens. When the throughput is maximized at $s \approx 120 \mu \mathrm{m}$, the lens is imaging with unity magnification, so the focal length may be found as $f \approx s / 4 \approx$ $30 \mu \mathrm{m}$. Under these conditions, the fiber-lens-fiber coupling loss was measured as $-5.9 \mathrm{~dB}$ in comparison to a fiber-fiber butt joint. At $\lambda=1.523 \mu \mathrm{m}$, the coupling loss reduced to -3.6 dB.

The expected optical properties have been estimated from a model that ignores the finite width of the circumferential land, assumes similar radii for the two spherical surfaces, and includes shrinkage. With these assumptions, the final radius $r$ of the surfaces is related to the initial resist diameter $w$ and height $h$, and the silicon frame thickness $t$, by

$$
2 V=\alpha \pi w^{2} h / 4, \quad \text { where } \alpha=\eta_{v}+\left(\eta_{v}-1\right)(t / h)
$$

Equations (1) and (2) may be solved numerically, and the focal length found using the lens-maker's formula $f=r /\{2(n-$ 1) $\}$ where $n$ is the refractive index of the resist. Assuming that $n \approx 1.5$, the theoretical focal length of the 40 - $\mu$ m-diameter lens 


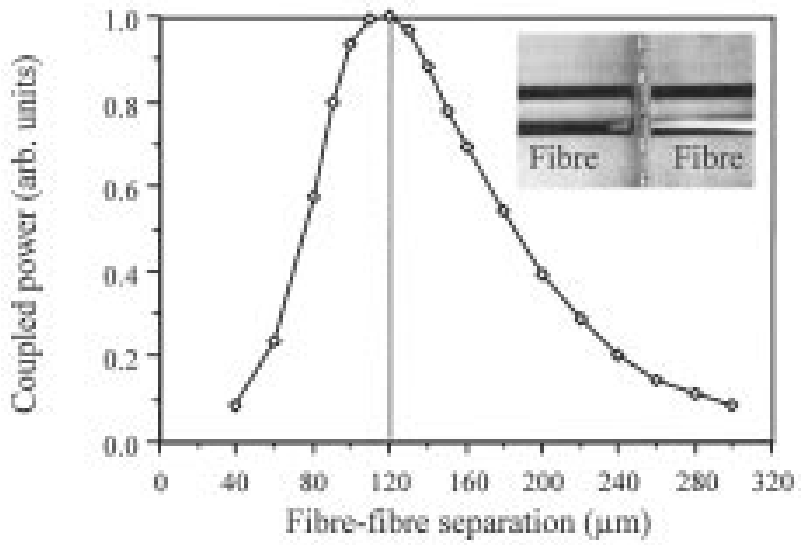

Fig. 5. Variation of throughput with separation in fiber-lens-fiber coupling experiment.

above is $f \approx 30.7 \mu \mathrm{m}$, in good agreement with the experimental measurement above.

Since the diameter and focal length of the lenses above are small, we now consider the modifications needed for more realistic values. When $\theta$ (and the numerical aperture) is small, Equation (1) reduces to $V \approx \pi w^{4} / 64 r$. The radius, focal length, and NA are then

$$
r \approx w^{2} /(8 \alpha h), \quad f \approx w^{2} /\{16 \alpha h(n-1)\}
$$

and

$$
\mathrm{NA} \approx 8 \alpha h(n-1) / w .
$$

Fig. 6 shows the variation of focal length and numerical aperture with initial pad diameter w obtained from Equation (3), assuming that $h=12 \mu \mathrm{m}, t=5 \mu \mathrm{m}$, and $\eta_{v}=0.75$ (so that $\alpha=0.646$ ). The focal length rises and the NA drops as $w$ increases. A diameter pad $w=200 \mu \mathrm{m}$ gives $f \approx 650 \mu \mathrm{m}$ and $\mathrm{NA} \approx 0.15$, which would be suitable for collimating the output of single mode fibers on a $250-\mu \mathrm{m}$ ribbon pitch. The main difficulty in fabricating such large lenses would lie in undercutting during sacrificial etching.

In conclusion, we have demonstrated the use of surface tension powered self-assembly to construct a three-dimensional

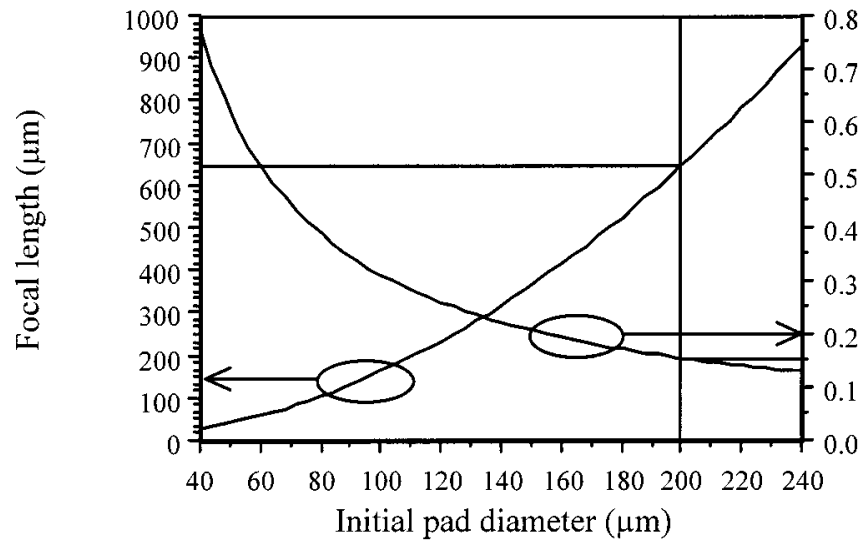

Fig. 6. Predicted variation of focal length and numerical aperture with initial pad diameter.

silicon frame carrying collimating microlenses formed by reflow molding of photoresist. The supporting frame is assembled accurately, and the resulting lenses have predictable properties when resist shrinkage is taken into account.

\section{ACKNOWLEDGMENT}

The author is very grateful to BCO Technologies (NI) Ltd. for manufacturing the BSOI material and performing the Si etching.

\section{REFERENCES}

[1] M. C. Wu, "Micromachining for optical and optoelectronic systems," Proc. IEEE, vol. 85, pp. 1833-1856, Nov. 1997.

[2] L. Y. Lin, S. S. Lee, K. S. J. Pister, and M. C. Wu, "Three-dimensional micro-Fresnel optical elements fabricated by micromachining techniques," Elect. Lett., vol. 30, pp. 448-449, 1994.

[3] C. R. King, L. Y. Lin, and M. C. Wu, "Out-of-plane refractive microlens fabricated by surface micromachining," IEEE Photon. Technol. Lett., vol. 8, pp. 1349-1351, Oct. 1996.

[4] J. S. Legatt and M. C. Hutley, "Microlens arrays for interconnection of singlemode fiber arrays," Elect. Lett., vol. 27, pp. 238-240, 1991.

[5] R. R. A. Syms and E. M. Yeatman, "Self-assembly of fully three-dimensional micro-structures using rotation by surface tension forces," Elect. Lett., vol. 29, pp. 662-664, 1993.

[6] R. R. A. Syms, "Surface tension powered self-assembly of 3-D micro-optomechanical structures," IEEE/ASME J. Microelectromech. Syst., vol. 8, pp. 448-455, 1999. 